\title{
Frequency-Coded mm-Wave Tags for Self-Localization System Using Dielectric Resonators
}

\section{A. Jiménez-Sáez ${ }^{1}$ (D) A. Alhaj-Abbas ${ }^{2} \cdot$ M. Schüßler ${ }^{1}$ - A. Abuelhaija ${ }^{2,3} \cdot$ M. El-Absi ${ }^{2}$. M. Sakaki ${ }^{4}$ L. Samfaß ${ }^{5} \cdot$ N. Benson ${ }^{4} \cdot$ M. Hoffmann ${ }^{5} \cdot$ R. Jakoby ${ }^{1} \cdot$ T. Kaiser ${ }^{2}$ • K. Solbach ${ }^{2}$}

Received: 20 March 2020 / Accepted: 18 May 2020 / Published online: 24 June 2020

(C) The Author(s) 2020

\begin{abstract}
The paper describes the development of passive, chipless tags for a novel indoor self-localization system operating at high mm-wave frequencies. One tag concept is based on the low-Q fundamental mode of dielectric resonators (DR) which exhibits peak scattering at its resonance frequency. As the radar cross-section (RCS) of DRs at mm-wave frequencies is far too low for the intended application, arrays of DRs and combinations with dielectric lens and corner reflectors are investigated to boost the RCS while keeping the scattering retro-directive over wide-angle incidence. Satisfactory results are demonstrated experimentally in Wband with metal corner reflectors combined with planar arrays of DRs; the tags produce a high RCS level over a moderately broad angular range and a wide frequency range where they exhibit a notch at the resonance frequency of the dielectric resonators. These designs suffer from low coding range of 3 to 6 bit, degradations of RCS in angular range, and a difficult separation of the tag response from strong clutter. Both the suppression of large clutter interference by using time gating of the tag response and a larger coding range are promised by a chipless tag concept based on multiple high-Q resonators in photonic crystal $(\mathrm{PhC})$ technology. Experimental samples are characterized as transmission resonators and as retro-directive tags at the $230 \mathrm{GHz}$ band. As a concept to boost the retro-directive RCS with a truly wide-angle response, the integration of $\mathrm{PhC}$ resonators with a Luneburg lens is discussed.
\end{abstract}

Keywords Radar cross-section · Photonic crystal - Dielectric resonator · Corner reflector . Luneburg Lens · Tag

\section{A. Jiménez-Sáez}

aljimse@gmail.com

Extended author information available on the last page of the article 


\section{Introduction}

Automation in logistics and industrial processes increasingly requires highly precise, autarch self-localization of, e.g., robots or autonomous vehicles in an indoor environment. While outdoor localization and positioning systems are dominated by satellite-based radio technology (GPS, etc.), modern indoor positioning systems can use a wide range of positioning technologies [1], from magnetic field based to optical and vision based to systems employing existing wireless infrastructure, like Bluetooth and Wi-Fi [2]. The latter systems employ the lower microwave spectrum and thus are limited to localization accuracies in the cm-range; even the high microwave frequencies in $5 \mathrm{G}$ systems will not provide below-cm accuracies [3]. Of the wide choice of alternative technologies, the optical, laser-based systems stand out by their potential to provide highly accurate position information, e.g., [4]. However, with the recent advances in monolithically integrated circuit solutions for mm-wave FMCW frontends [5, 6], a potential competitor technology arises: Highly integrated Radar modules are attractive as reader frontends (with antennas included) for a self-localization system due to their low-cost, light-weight construction, and low power consumption. Most importantly, the achievable large sweep bandwidths of more than $30 \mathrm{GHz}$ at high mm-wave frequencies in combination with the coherent signal processing of such FMCW transceivers allow sub-mm range resolution; in addition, transmit power combining can be employed to increase range and phased array techniques can be employed to realize inertia-less beam control. In our cooperative research center, we combine research in the FMCW Radar microelectronic technology and Radar system design with the research on applications like an indoor self-localization system. To achieve sub-mm localization accuracies at several meter read range, we proposed a novel selflocalization system [7] which is intended to work at high mm-wave frequencies (or THz); this system employs passive, chipless tags as landmarks fixed to the infrastructure and a wide-band FMCW Radar as the reader.

Inspired by the use of dielectric resonators (DR) as passive tags for RFID and sensing applications by Mandel et al. [8], our original proposal employs DRs as tags to provide retrodirective monostatic radar cross-section (RCS) with peak levels at the DR resonance frequencies. This way, each tag can be discriminated by its frequency signature (frequency coding) and the high-level return signal around the resonance frequency can be used for precise ranging. Figure 1 gives a sketch of the considered system, where a Radar reader transmits a swept frequency signal and scans its environment by the antenna beam. The room is equipped with retro-directive chipless tags functioning as landmarks with precisely known positions $\left(X_{\mathrm{Tm}}, Y_{\mathrm{Tm}}\right)$. After the reader has detected, identified (by resonant frequency), and ranged the landmarks in its environment, its own position coordinates $\left(X_{\text {Reader }}, Y_{\text {Reader }}\right)$ can be calculated from the ranges to the landmarks and the knowledge of the landmark positions.

High RCS of the chipless tags over a broad incident angle is required to achieve precise ranging by the Radar reader. However, even the corners of a room or some furniture create corner reflectors that might as well generate high-level reflections and, in some cases, arrive at the reader with a similar angle-of-arrival and at a nearby time so that the readout of the tag response is not possible. This situation is sketched in Fig. 1b, where the received signal of a mobile reader is plotted for a short pulse interrogation of tags of different quality factors. Note that the tag frequency coding is contained in the ringing tail of the scattered pulse and that for a tag of low $Q$-factor $(Q=50)$ the close-by clutter pulses can completely cover this part of the tag response; in this case, the tag frequency coding cannot be resolved. A solution to this problem was proposed in our 

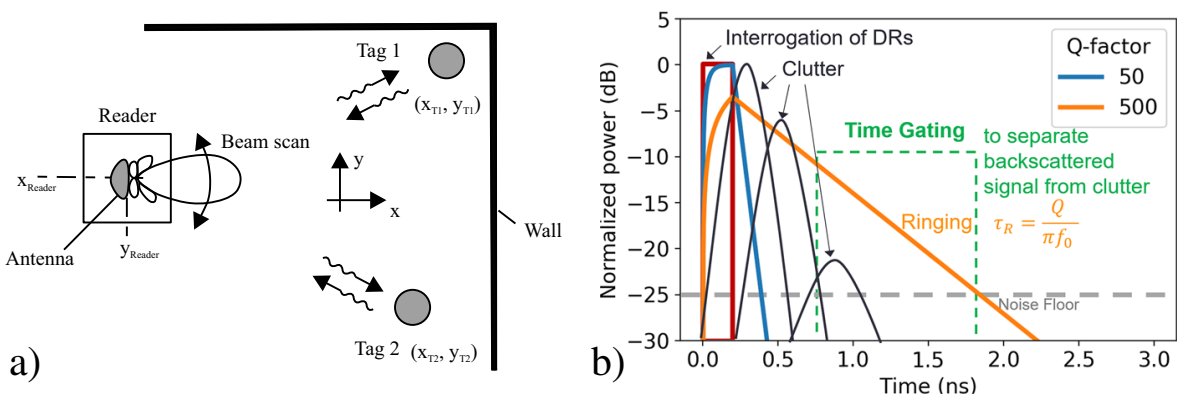

Fig. 1 a Sketch of the indoor self-localization system setup. b Effect of the quality factor on the charge and discharge time of two different resonators with quality factors of 50 and 500 when excited by a 0.2 ns pulse at $230 \mathrm{GHz}$. Some reflections from nearby clutter are sketched. The higher the quality factor, the more flexibility in order to suppress clutter with time gating

early concept paper [9]: If we employ a high-Q resonant mode with long ringing time, a Radar reader can identify the frequency coding by using a time gate to cut-out the latetime ringing after the clutter responses have died out. This is indicated in Fig. 1b where the time gate covers the time after two large clutter pulses until the ringing signal level crosses the noise floor. In microwave circuit technology, it is quite common to create electro-magnetically screened filter networks or oscillator circuits using dielectric resonator modes with Q-factors of several thousand. However, as soon as we operate the DRs in free space, radiation losses reduce the Q-factors to about fifty for fundamental modes up to several hundred for higher order modes. As already reported in [9], far above the fundamental mode frequency, the spectrum is closely filled with interfering resonant modes which very much limits the practicability of this approach. We therefore discarded the concept of using higher order DR modes in the landmark tags. Instead, we took two different approaches to solve the problem: On the one hand, we considered replacing the discrete (higher order mode) DRs by resonators based on an alternative technology. We decided to investigate photonic crystals $(\mathrm{PhC})$ for the creation of high-Q resonators suitable for a later implementation as retro-directive tags of moderately high RCS level (for the compensation of amplitude decay by the late-time ringing). On the other hand, we reassessed the clutter problem of tag detection using discrete DRs. We concluded that even a DR tag of low-Q, i.e., a fundamental mode resonator should remain visible in clutter, if we could boost its RCS so that it was larger than the clutter level. So, initially, our research was directed towards $\mathrm{PhC}$ high-Q resonator tags on the one hand and towards DR fundamental mode high-RCS tags on the other hand. Based on the results of these investigations, a combination of approaches seems to be feasible which could satisfy the system requirements indicated above.

In the following Section 2, we give an account of the development of fundamental mode DR tags with the aim to combine wide-angle retro-directive high RCS with clear signature for identification. For illustration, detailed experimental results of some realized tags are presented, using a W-band Vector Network Analyzer (VNA); in one example, we also show the measurement result from an FMCW Radar to demonstrate its feasibility as a reader. Drawbacks of the fundamental mode tag design are found to be avoided by the concept of high-Q retro-reflective tags. The development of multi-resonator high-Q resonator tags is described in Section 3 and experimental results at 220 to $330 \mathrm{GHz}$ are presented. In Section 4, we conclude the paper. 


\section{Towards Low-Q/High-RCS Resonator Tags}

Our pragmatic approach of enabling the reader to detect, range, and identify a landmark in many situations without long ringing times is to employ tags with wide-angle retrodirective RCS which is high enough to top the clutter; based on preliminary lab experiments at W-band, we assume the required RCS levels on the order of + $10 \mathrm{dBm}^{2}$. For easier experimentation and due to better availability of DRs, our initial investigations used DRs with fundamental mode resonance frequencies in the $5-10 \mathrm{GHz}$ range. At microwave frequencies, low-loss ceramic resonators are available from many sources, but this is no longer the case when we require much smaller resonators for $\mathrm{mm}$ wave frequencies. Instead of expensive custom fabrication of such resonators, we found satisfactory mm-wave performance in spheres of $\mathrm{ZrO}_{2}$ made for ball bearings and such balls of 0.6 and $0.5 \mathrm{~mm}$ diameter were used in the tag designs for W-band. In the following, various tag designs are discussed and summarized in Table 1.

Even at microwave frequencies, RCS levels of single DRs are found very low [10] and scaling to mm-wave frequencies further reduces the RCS levels by the square of the frequency ratio: As an example, in Fig. 2a, we show the field distribution and bi-static RCS pattern of a single spherical DR of $0.5 \mathrm{~mm}$ diameter with its fundamental $\mathrm{TE}_{101}$ mode [11] resonant at $103 \mathrm{GHz}$; the next higher mode appears at $1.4 \times 103 \mathrm{GHz}$. Due to the DR symmetry, the mode fields are excited by the incident wave such that the scattering is retrodirective. In Fig. 2b, we show a simulation of the monostatic RCS signatures together with measurement results for DRs of $0.5 \mathrm{~mm}$ and $0.6 \mathrm{~mm}$ diameter. Note that the peak RCS level of the smaller DR is about $-52 \mathrm{dBm}^{2}$ at resonance. By choosing different sizes of DRs, tags could be discriminated; due to the resonance bandwidth seen in the signatures, a separation of about 3 to $5 \mathrm{GHz}$ should be kept to avoid overlapping, i.e., about 10 different frequency positions could be used or "coded" in individual tags (equivalent to a 3-bit code) covering

Table 1 Summary of experimental tag designs

\begin{tabular}{|c|c|c|c|}
\hline Type & Dimensions & Spectral signature & Approx. RCS \\
\hline PEC sphere & $15 \mathrm{~mm}$ dia. & Flat & $-37 \mathrm{dBm}^{2} @ 100 \mathrm{GHz}$ \\
\hline Single DR & $\begin{array}{l}1 \times 0.6 \mathrm{~mm} \text { dia. } \\
1 \times 0.5 \mathrm{~mm} \text { dia }\end{array}$ & Single resonant peak & $\begin{array}{l}-50 \mathrm{dBm}^{2} @ 86 \mathrm{GHz} \\
-52 \mathrm{dBm}^{2} @ 105 \mathrm{GHz}\end{array}$ \\
\hline Diel. lens and DR & $\begin{array}{l}\text { Lens } 6 \mathrm{~mm} \text { dia. } \\
\text { DR } 0.5 \mathrm{~mm} \text { dia. }\end{array}$ & Single resonant peak & $-22 \mathrm{dBm}^{2} @ 105 \mathrm{GHz}$ \\
\hline Lin. array of DRs & $\begin{array}{l}9 \times \text { DR } 0.6 \mathrm{~mm} \\
11 \times \text { DR } 0.5 \mathrm{~mm}\end{array}$ & Double resonant peak & $\begin{array}{l}-28 \mathrm{dBm}^{2} @ 86 \mathrm{GHz} \\
-30 \mathrm{dBm}^{2} @ 105 \mathrm{GHz}\end{array}$ \\
\hline Planar array of DRs & $\begin{array}{l}9 \times 5 \mathrm{DRs} 0.6 \mathrm{~mm} \text { dia. } \\
25 \mathrm{~mm} \times 15 \mathrm{~mm}\end{array}$ & Single resonant peak & -11 dBm² @84 GHz \\
\hline Lin. array of DRs over flat plate & $\begin{array}{l}9 \times \text { DRs } 0.6 \mathrm{~mm} \text { dia. } \\
10 \mathrm{~mm} \times 25 \mathrm{~mm}\end{array}$ & Single notch & -10 dBm²@ @-band \\
\hline Planar array of DRs over flat plate & $\begin{array}{l}9 \times 5 \text { DRs } 0.6 \mathrm{~mm} \text { dia. } \\
28 \mathrm{~mm} \times 20 \mathrm{~mm}\end{array}$ & Single notch & -8 dBm²@W-band \\
\hline Lin. array of DRs in corner reflector & $\begin{array}{l}7 \times \mathrm{DR} 0.4 \mathrm{~mm} \mathrm{Al}_{2} \mathrm{O}_{3} \\
2 \times 7 \mathrm{~mm} \times 10 \mathrm{~mm}\end{array}$ & Single notch & $-14 \mathrm{dBm}^{2} @ 230 \mathrm{GHz}$ \\
\hline $\begin{array}{l}\text { Planar array of DRs on dihedral } \\
\text { corner reflector plates }\end{array}$ & $\begin{array}{l}7 \times 7 \text { DRs } 0.5 \mathrm{~mm} \text { dia. } \\
5 \times 5 \text { DRs } 0.6 \mathrm{~mm} \text { dia. } \\
2 \times 10 \mathrm{~mm} \times 10 \mathrm{~mm}\end{array}$ & Double notch & -6 dBm²@W-band \\
\hline $\begin{array}{l}\text { Planar array of DRs in trihedral } \\
\text { corner reflector aperture }\end{array}$ & $\begin{array}{l}158 \mathrm{DRs} 0.6 \mathrm{~mm} \text { dia. } \\
\text { in } 2.5 \mathrm{~mm} \text { grid } \\
68 \mathrm{~mm} \text { edge length }\end{array}$ & Single notch & +8 dBm²@W-band \\
\hline
\end{tabular}


a)
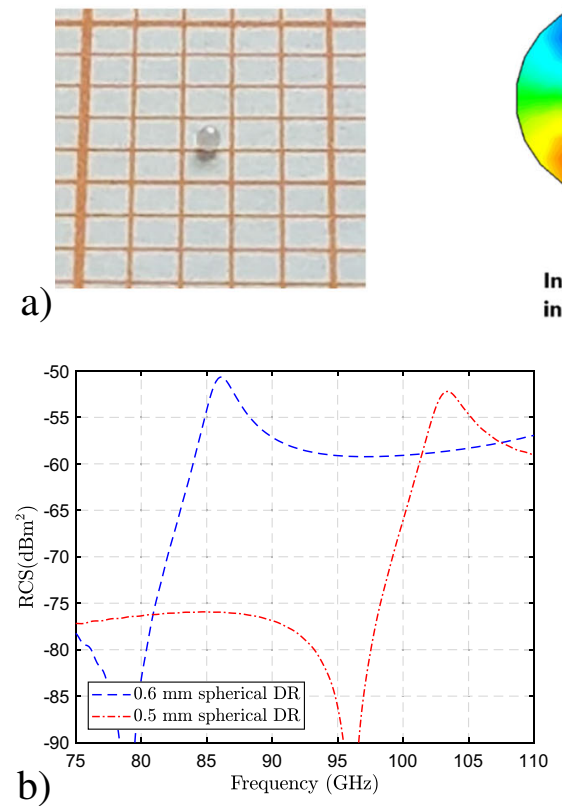
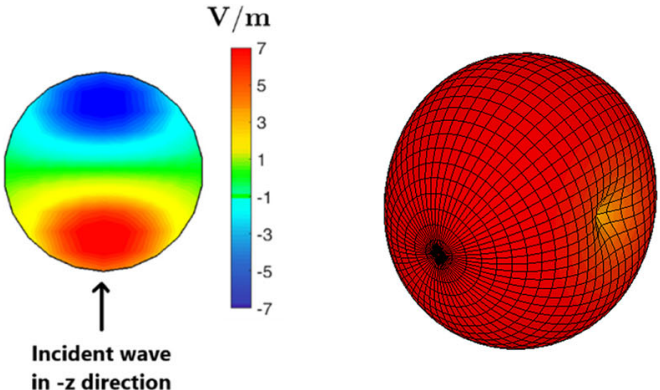

in $-z$ direction

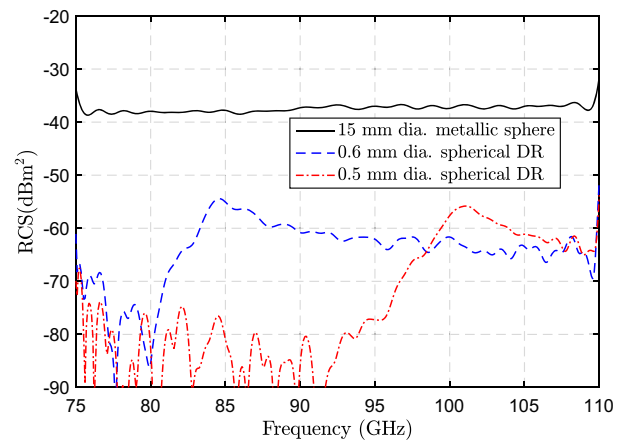

Fig. 2 a Picture of single spherical $\mathrm{ZrO}_{2}$ resonator on millimeter plotting paper and simulated field distribution of the TE101 mode and its bi-static RCS pattern (rel. permittivity of 32 and simulated with CST Microwave Studio). b Simulated RCS signatures of DRs of $0.5 \mathrm{~mm}$ and $0.6 \mathrm{~mm}$ diameter compared with measured signatures of DRs; measured reflection coefficients were normalized to RCS levels using a reference measurement of a $15-\mathrm{mm}$ steel ball (RCS approx. $-37 \mathrm{dBm}^{2}$ at band center). DUTs placed in $36 \mathrm{~cm}$ distance to horn antenna

the fundamental mode frequency range (1:1.4 or $33 \%$ bandwidth). The observed bandwidths correspond to Q-factors of the resonances of about 30-50 which result from radiation loss rather than dielectric dissipation losses (see the discussion of loss-factors in Section 3). RCS spectral signatures are proportional to the reflection coefficient which can be measured in front of a horn antenna: Most of the laboratory measurements in this section are made using a R\&S ZVA67 Vector Network Analyzer driving the W-band extender ZC110 which connects to a $25 \mathrm{dBi}$ horn antenna. To increase accuracy and suppress interactions of test object (DUT) and horn antenna, we first perform a waveguide calibration, transform to time domain, isolate the DUT on its rotatable Styrofoam support by time gating, and retransform to frequency domain. Finally, plots are taken after empty room subtraction.

In our early work at microwave frequencies, the first approach to boost the RCS levels and still maintain wide-angle retro-directivity was a combination of DRs with spherical lenses [12, 13] or $2 \mathrm{D}$ lenses $[14,15]$ and we even realized tags with angle-of-arrival sensing [16]. Feasibility of the lens combination for mm-waves was proven using a Polyamid sphere of $6 \mathrm{~mm}$ diameter with a spherical DR of $0.5 \mathrm{~mm}$ diameter glued to a paper support behind the lens. This design gave a signature of the RCS with the resonance peak increased by about $30 \mathrm{~dB}$ over the response by the spherical DR alone. By simulation, we find that the lens focuses the incident wave towards the DRs sitting close to the lens periphery and reflecting the incident wave at the $\mathrm{TE}_{101}$ mode resonance frequency. To make the lens-DR combination effective over a wide range of incident angle, several DRs have to be placed along the focal circle [13]. 
It was noted that the increase in RCS by use of dielectric lenses is limited to about $30 \mathrm{~dB}$ due to the structural reflections from the $3 \mathrm{D}$ dielectric lenses and the metallic parts of the investigated 2D lenses. However, much larger spherical Luneburg lenses may be employed with much higher gain in RCS; this is found to be due to much lower structural scattering [12] because of the progressive gradient of the refraction index. We verified this using a large spherical Luneburg lens for use at the $10 \mathrm{GHz}$ frequency range produced by 3D printing (polymer jetting). Recently, with advanced 3D printing technology, designs of Luneburg lenses for W-band have been realized [17], which should allow a drastic increase in RCS and in the readout range of single resonators even at high mm-wave frequencies.

As an alternative method to produce high retro-directive scattering levels, arrays of DRs can be used $[18,19]$ and such tag landmarks have been employed successfully in a localization system demonstrator at the microwave frequency range [20]. As an example, we tested a double column array of 9 resonators of $0.6 \mathrm{~mm}$ and 11 resonators of $0.5 \mathrm{~mm}$ diameter. The DRs were bonded to one common carrier (half-cut plastic straw of $0.1 \mathrm{~mm}$ thickness and $20 \mathrm{~mm}$ height stuck into a triangular piece of Rohacell foam) by narrow strips of double-sided adhesive tape (product name tesa). The two arrays are closely spaced by $5 \mathrm{~mm}$ (diameter of the plastic straw) such that coherent superposition of scattering contributions creates strong variation of the RCS signature with the angle of incidence. Compared with a single DR, the RCS is boosted by about $20 \mathrm{~dB}$.

A second example presents a planar array of $9 \times 5$ DRs of $0.6 \mathrm{~mm}$ diameter bonded to a small sheet of paper by a double-sided adhesive tape (product name tesa) (Fig. 3). Due to the paper and tape, at normal incidence $\left(0^{\circ}\right)$, we observe high scattering level over the band with only $5 \mathrm{~dB}$ increase due to DR resonance. This demonstrates that it is important to create a support structure which has very low structural reflections and still can fix the DRs in a precise planar arrangement. At higher incidence angles, retro-directive scattering reduces due to specular forward reflection. However, due to the element grid of 0.7 wavelength $(2.5 \mathrm{~mm}$ at $85 \mathrm{GHz}$ ), we observe a grating lobe at $45^{\circ}$ incidence angle; to avoid this, we either have to bring the DRs closer together or rotate the planar array by $45^{\circ}$ such that the plane of incidence is an intercardinal plane.

From a system perspective, the crucial problem of large linear and planar arrays of DRs turns out to be the limitation of the high retro-directive RCS only close to the direction of incidence which is normal to the axis of a linear array or broadside to a planar array. In addition, fabrication tolerances become more and more challenging to ensure that all


Fig. 3 Planar array of $9 \times 5$ DRs of $0.6 \mathrm{~mm}$ diameter in $2.5 \mathrm{~mm}$ pitch bonded to a paper support and measured RCS signatures for incidence angle from $0^{\circ}$ (normal) to $45^{\circ}$. DUT placed in $75 \mathrm{~cm}$ distance to horn antenna 
resonators have the same resonance frequency to respond coherently when the dimensions are scaled down to operate at $\mathrm{mm}$-wave or even $\mathrm{THz}$ frequencies.

Another important limitation which is common to all the approaches mentioned above: When it comes to the range processing by an FMCW Radar reader, the wide frequency sweep range cannot provide the full expected range resolution and accuracy because the effective target response is limited to the narrow bandwidth around the resonance frequency, e.g., $5 \mathrm{GHz}$ at $\mathrm{W}$-band. To overcome this limitation, our investigations turned to combinations of DRs with large metal reflectors which can provide high-level RCS over the full reader bandwidth. Surprisingly, the spectral signature of such combinations was found inverse to the signatures of the pure DR tags: Instead of a peak RCS at resonance, we observed a notch in the broadband high-level RCS signature of the metal reflectors. The "mechanism" of this was found to be scattering by the DRs and not absorption which can be shown by simulations with lossless DRs. Investigations started with a single DR and a linear array of DRs in front of a flat metal plate [21]. As an example, Fig. 4 shows a column array of 9 DRs sitting on a foam adhesive pad (product name Strips Pattex HS PXMS1) of $1 \mathrm{~mm}$ height on the surface of a flat metal reflector. The comparison to the signature of the flat metal surface without DRs (backside, $\theta=180^{\circ}$ ) shows very little loss (below $1 \mathrm{~dB}$ ); the superposition of the DR reflections and the reflections from the metal part produce varying signatures depending on the angle of incidence. This tag design was also evaluated using an FMCW Radar [22]; the measured signature compares well which proves that we can use such a Radar as the reader in our localization system. The notch depth seen in the measurement for normal incidence $\left(0^{\circ}\right)$ reduces when the width of the reflector is increased so that this tag design is limited in the achievable RCS level. However, notch depth can be kept high for larger reflector surfaces and thus for higher RCS levels by using a planar array in front of a metal plate instead of a linear array. This was proven by using the planar array of Fig. 4 in front of a flat metal reflector plate of $28 \mathrm{~mm} \times 20 \mathrm{~mm}$. From the comparison of the reflection magnitudes with and without the planar array, we concluded that the insertion loss of the DR array with paper and adhesive tape support structure is below $1 \mathrm{~dB}$. It is also found that, for optimum notch depth, the DRs must be placed at a distance from the metal reflector of approximately half-wavelength or multiples.

These designs combine high RCS level with a useful frequency position coding by a notch signature; however, they are still not satisfactory as they also are limited in incidence angle to the near-broadside directions.


Fig. 4 A linear array of $9 \mathrm{DRs}$ of $0.6 \mathrm{~mm}$ diameter fixed to a quadratic metal tube with a flat reflector surface of $10 \mathrm{~mm} \times 25 \mathrm{~mm}$ by double-sided foam adhesive strip. Left plot: RCS spectral signature at normal incidence $\left(0^{\circ}\right)$,

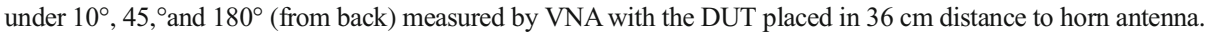
Right plot: Spectral signature measurements of VNA compared with FMCW Radar [22] 
Finally, the required wide-angle performance was realized by adopting the concept of the corner reflector instead of flat metal reflectors. In a first step, column arrays of DRs were placed in front of corner reflectors [21]. However, like in the case of the linear array in front of a flat metal reflector, this approach is also limited because the width dimension is limited; in the case of a realization for $230 \mathrm{GHz}$ notch frequency, the width of the dihedral corner reflector could not be increased above $10 \mathrm{~mm}$ without reducing the notch depth to below $3 \mathrm{~dB}$. Therefore, in a second step, we progressed to planar arrays placed in front of the plates of a corner reflector. Theory and experimental proof at microwave frequencies is given in [23]. In one example at W-band, [24], we used a dihedral corner reflector of two plates of $20 \mathrm{~mm} \times$ $20 \mathrm{~mm}$ size with one planar array of DRs attached to each plate; one array used $7 \times 7$ DRs of $0.5 \mathrm{~mm}$ diameter and the other array used $5 \times 5 \mathrm{DRs}$ of $0.6 \mathrm{~mm}$ diameter. The resulting RCS spectral signature exhibits one notch at $83 \mathrm{GHz}$ and one notch at $100 \mathrm{GHz}$ due to the different DR sizes. This design allows corner reflector sizes as large as required to overcome the clutter while providing its high RCS and deep notch signature over a wide range of incidence angle. Additionally, as in this example, the freedom to place the two distinct spectral notch positions by choosing suitable DR sizes increases the frequency position coding range for tag identification to equivalent 5-bit over the equivalent 3-bit when only a single DR size is used. The planar DR array concept for the dihedral corner reflector may also be applied to the trihedral reflector with three DR arrays and possibly three different DR sizes for an even larger coding range equivalent to 6-bit.

A simpler and very efficient alternative to the above corner reflector design was found by adopting a concept [25], first demonstrated using a stopband frequency selective surface (FSS) based on conductor patterns (crossed dipoles) as depicted in Fig. 5. The FSS is placed in front of the aperture of a corner reflector so that the incident wave is reflected away from the corner reflector at the stopband frequency and a notch in the RCS spectral signature is created. With a $3 \times 3 \times 3 \mathrm{~cm}^{3}$ trihedral corner reflector and an FSS in front of it, ranges up to $4 \mathrm{~m}$ were demonstrated without "empty room" channel subtraction using a Vector Network Analyzer Agilent Technologies N5222A with an Anritsu 3740A W-Band extension and a $25 \mathrm{dBi}$ reader antenna.

We modify this design by replacing the printed dipole FSS by a planar array of DRs in front of a trihedral corner reflector (Fig. 6): The array of DRs acts as reflecting surface for an incident wave with a peak reflection at around the resonant frequency of the DRs; by this, a
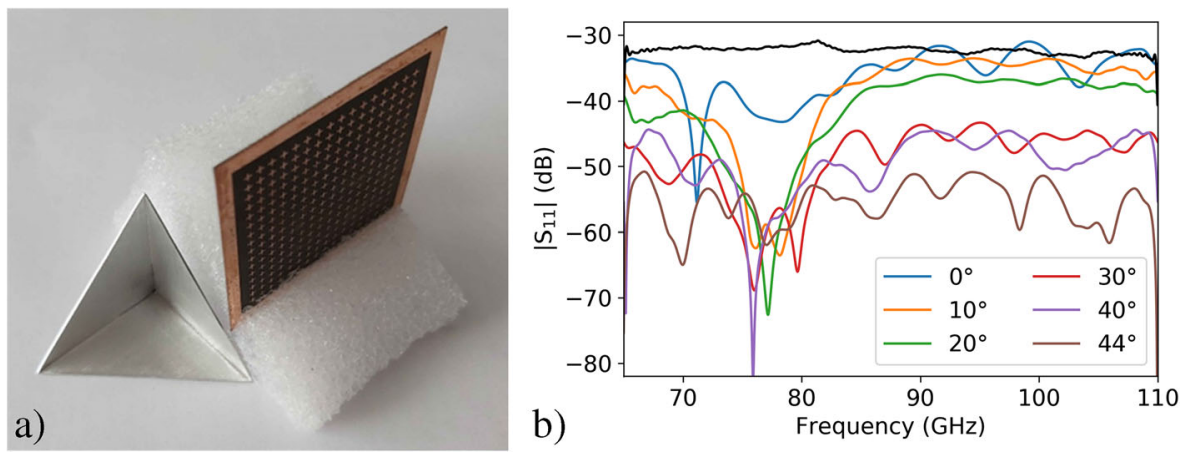

Fig. 5 a Trihedral corner reflector with FSS cover and $\mathbf{b}$ the measured RCS signatures at a distance of $50 \mathrm{~cm}$ for TM polarization. In black, the response of an uncoded trihedral corner reflector for $0^{\circ}$ incidence is shown as a reference [25] 

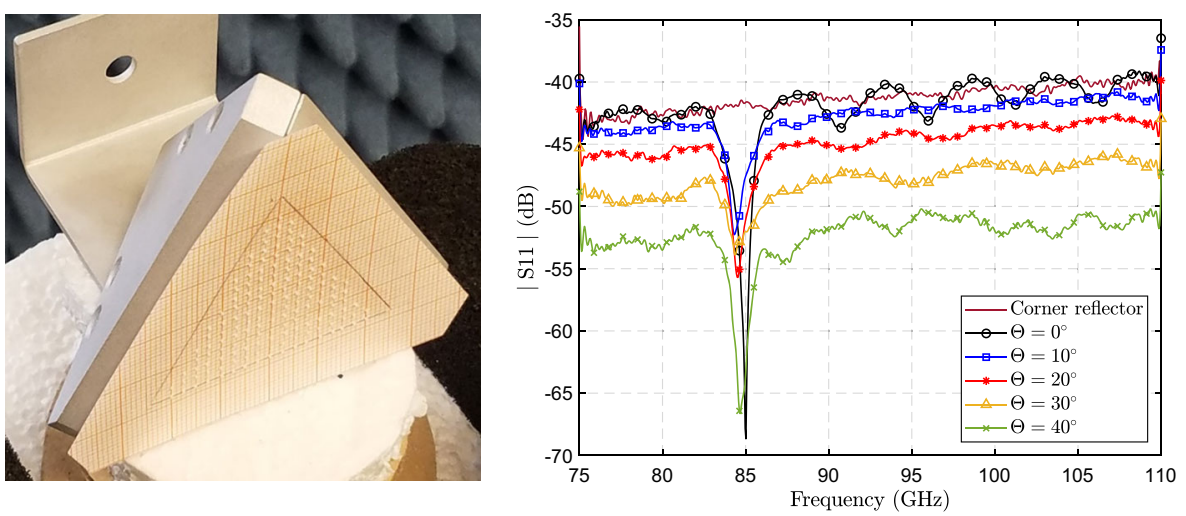

Fig. 6 Trihedral corner reflector of $68 \mathrm{~mm}$ edge length with planar array of 158 DRs of $0.6 \mathrm{~mm}$ diameter in a 2.5-mm grid fixed to the corner reflector aperture. Measured RCS spectral signatures for incident angle from $0^{\circ}$ to $40^{\circ}$ with signature of the reflector without DR array (full line) for comparison; DUT placed in $2 \mathrm{~m}$ distance to horn antenna

large part of the incident wave is reflected away from the corner reflector and the resulting RCS drops to create a notch in the spectral signature. Outside the resonance regime, the DRs let the incident wave pass to the corner reflector which produces strong retro-directive RCS over the rest of the frequency band. We see a resonant notch at about $85 \mathrm{GHz}$ which varies in depth with the angle of incidence. Outside the resonance region, the RCS level behaves similar to the corner reflector without the DR array, i.e., reducing RCS levels with increasing incidence angle; at normal incidence $\left(0^{\circ}\right)$, we observe a ripple of about $\pm 1.5 \mathrm{~dB}$ which results from internal reflections between the corner reflector and the planar DR array. To keep a flat signature at normal incidence, the reflections from the planar DR array should be kept low by employing a very thin support structure of low permittivity; in our example, we used a paper of $0.9 \mathrm{~mm}$ thickness with a $10 \mu \mathrm{m}$ thick layer of glue (product name UHU POR) for bonding the DRs. For a distinct notch signature at resonance, the distance from the aperture plane of the corner reflector apex has to be chosen such that the superposition of the reflections from the corner reflector and from the DR array superimposes destructively at the DR resonance frequency; in our example, the DR array was fixed to the edges of the corner reflector without adjustment. The tag shown in Fig. 6 employs a metal corner reflector with a triangular aperture of $68 \mathrm{~mm}$ edge length with a planar DR array covering about only half the area of the reflector aperture (158 resonators inside the inscribed triangle of $50 \mathrm{~mm}$ side length). More DRs may be placed on the array plane to improve the notch depth but a smaller number of DRs reduces the notch depth: for example, with $45 \mathrm{DRs}$, the notch depth reduced to only about $3 \mathrm{~dB}$.

From the summary of the above discussed tag designs (Table 1), it becomes clear that regarding the target specification of our "low-Q/high-RCS" landmark tag, the last corner reflector examples appear quite attractive. This is because the corner reflector sizes (and by this, the RCS) can be made as large as required to overcome the clutter, while providing its high RCS over a wide range of incidence angle, and providing clear identification by the notch position in its spectral signature. The broadband high RCS level generated by the metal corner reflectors is highly appreciated, since this allows an FMCW Radar reader with wide sweep bandwidth, e.g., as used in [22], to create a very narrow target pulse. As an example, in Fig. 7, we show the impulse response generated by a frequency sweep of $35 \mathrm{GHz}$ from the reflection of the trihedral corner reflector of 
Fig. 7 Reflection impulse response of corner reflector of Fig. 6 at normal incidence. Dashed curve with DR array; full line curve without DR array

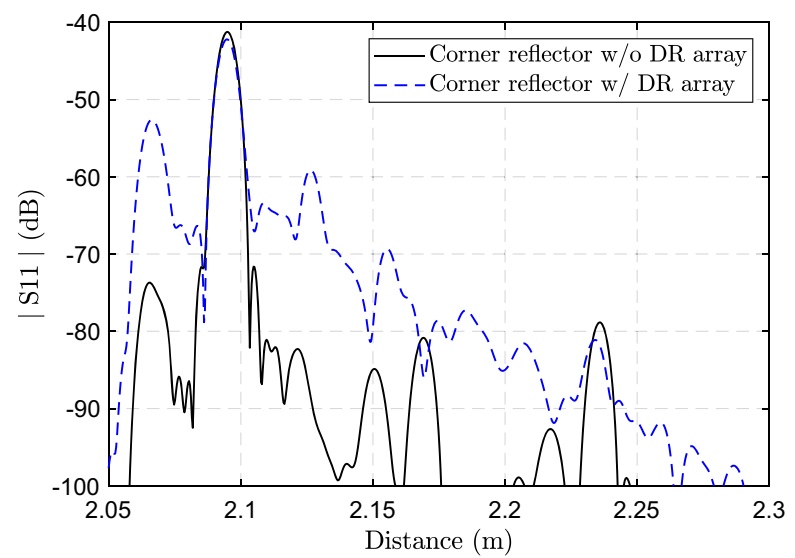

Fig. 6. The plot for the corner reflector without the DR array shows a first, low peak from the front edge of the metal corner reflector and a high main pulse from the trihedral reflector faces in about $3 \mathrm{~cm}$ distance, which is the depth of the corner reflector. The plot for the corner reflector with the DR array in place exhibits a first peak at the same position, but with $20 \mathrm{~dB}$ higher level due to the reflection from the DR array which is placed exactly across the metal reflector aperture. The main peak following this is still much larger and is due to the reflection from the corner reflector plates; after this peak, reflections between the planar array and the metal reflector produce peaks with decaying amplitude (standing wave effect) and a long ringing tail due to the excitation and discharge of the resonators is superimposed. The $-3 \mathrm{~dB}$ pulse width for the corner reflector peak is $0.04 \mathrm{~ns}$ which easily allows to range this landmark at resolution and accuracy in the sub-mm range while the landmark still can be identified by its spectral notch position. However, as discussed in Section 1, the ringing tail in the pulse shape may create a problem when the landmark sits close to a large infrastructure reflector, like a flat wall and the reader signal is normally incident to the wall: The superposition of a strong interferer pulse with the ringing tail of the landmark can critically distort the RCS notch in frequency domain and harm the tag identification.

With a tag providing much longer ringing time, the reader could gate-out the infrastructure clutter response and analyze the remaining ringing signal; with short ringing times, as in our example of Fig. 7, we have to keep a minimum distance to interfering infrastructure objects by selecting suitable installation positions. In addition, we may have to use some absorber shielding behind the reflector covering the first Fresnel zone and combine this with a suppression of the interfering pulse by a time gating in the signal processing of the reader.

There are, however, also certain limitations in the corner reflector tag design: As a result of the low-Q resonant modes, the number of non-overlapping notch positions is limited to only few equivalent bits; tags using narrow-band high-Q resonators could much relax this limitation. The other limitation concerns the typical RCS retro-directivity pattern of a corner reflector seen in Fig. 8. We observe a steep degradation of the RCS level with increasing incidence angle (and a peak around $40^{\circ}$ ) which severely could limit the read range of our system. As demonstrated in [13] and also indicated in Fig. 8, a spherical lens reflector could be an 
Fig. 8 Typical variation of retrodirective RCS as a function of incidence angle. Comparing a Luneburg lens reflector to a trihedral corner reflector

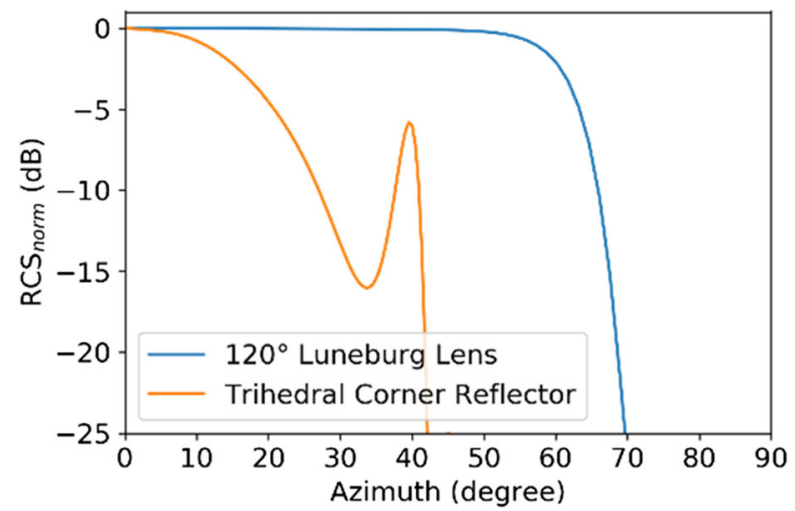

attractive alternative since it can provide a near-constant RCS level over a large angle range. As indicated above, both drawbacks of the corner reflector tag could be avoided by high-Q resonator tags which are presented next.

\section{Towards High-Q Resonator Tags}

The wireless readout of the resonance frequency of high-Q resonators has been demonstrated for sensing purposes below $10 \mathrm{GHz}$, showing how the long ringing response allows for their use in bridges [26], corridors [27], and high-reflective environments such as machine tools [28]. Such high-Q resonators may be realized with metallic or dielectric structures. For mmwave frequencies and above, the use of resonators based on a metallic cavity is often avoided due to metallic losses, which increment with the square root of the frequency and with the surface roughness of the metallic surface. In the case of dielectric resonators, the losses of the dielectric material $Q_{\mathrm{d}}$ represent an upper limit to the measured loaded quality factor $Q_{1}$ following the equations

$$
\frac{1}{Q_{1}}=\frac{1}{Q_{\mathrm{d}}}+\frac{1}{Q_{\mathrm{rad}}} \quad \text { and } \quad Q_{\mathrm{d}}=\frac{1}{\tan \delta_{\mathrm{d}}},
$$

where $Q_{\text {rad }}$ represents the radiation quality factor. In order to achieve high Q-factors over 500, low-loss materials with high relative permittivity such as $50[27,28]$ are employed.

As the frequency is increased towards $230 \mathrm{GHz}$, the availability of materials with highpermittivity and very low losses is reduced. The available materials that show the lowest dielectric loss with relative permittivity $\epsilon_{\mathrm{r}}>9$ are shown in Table 2 . Although $\mathrm{ZrO}_{2}$ presents low losses for several applications, these losses are too high for the realization of high-Q resonators even if just mixed with lower loss $\mathrm{Al}_{2} \mathrm{O}_{3}$ (alumina). In order to achieve high radiation quality factors $Q_{\mathrm{rad}}>500$, either high relative permittivity $\epsilon_{\mathrm{r}}$ or high-order resonance modes are needed. Materials with higher relative permittivity $\epsilon_{\mathrm{r}}>13$ and $\tan \delta<10^{-3}$ are unknown to the authors, while the use of higher order resonance modes results in higher mode density and therefore reduces the bandwidth available for the coding of information.

To overcome these limitations, photonic crystals ( $\mathrm{PhCs}$ ) can be used to reduce the radiation of dielectric resonators. By shifting the holes surrounding the resonator, cavities with radiation quality factors $Q_{\mathrm{rad}}>10^{6}$ can be realized [31]. Such resonators have been intensively studied in 
Table 2 High-permittivity materials

\begin{tabular}{llll}
\hline & $\mathrm{Al}_{2} \mathrm{O}_{3}$ & $\mathrm{HR}-\mathrm{Si}$ & $\begin{array}{l}20 \% \mathrm{ZrO}_{2} \\
80 \% \mathrm{Al}_{2} \mathrm{O}_{3}\end{array}$ \\
\hline$\epsilon_{\mathrm{r}}$ & & 11.68 & 11.28 \\
$Q_{\mathrm{d}}$ & $9 \ldots 9.9$ & $>2000$ & 500 \\
Frequency & 2940 & $200 \mathrm{GHz}$ & $144 \mathrm{GHz}$ \\
Reference & $144 \mathrm{GHz}$ & {$[30]$} & {$[29]$} \\
\hline
\end{tabular}

optics [32-34] and are being investigated in $\mathrm{THz}$ frequencies for the realization of highly sensitive sensors $[35,36]$. In addition to be able to modify the radiation properties of the resonators, the PhC structure serves as a mechanical support of these resonators while confining the EM energy in the resonators. Thus, $\mathrm{PhC}$ resonators can be mounted in lossy supports without affecting their resonant properties in the desired frequency band.

A verification of the low losses and the capability to design high-Q resonators with 3Dprinted alumina and deep reactive ion etched (DRIE) high-resistive silicon has been presented at $80 \mathrm{GHz}$ in [37]. After this successful characterization, the resonators with alumina and HRSi have been scaled to the 220 to $330 \mathrm{GHz}$ frequency range to fall under the WR3 output of the measurement equipment and the manufactured samples can be seen in Fig. 9. More information on the (DRIE) process for the fabrication of the HR-Si resonators and the lithographybased ceramic manufacturing (LCM) can be found in [37].

The measurement setup for the characterization of the high-Q resonators and the measured S-parameters is shown in Fig. 10. The resonators are mounted in a Polylactic Acid (PLA) support additively manufactured with a standard fused deposition modelling (FDM) process. Since the electromagnetic fields do not propagate to the borders of the $\mathrm{PhC}$ for the bandgap frequency range, the influence of the PLA support on the resonator frequency and measured quality factor can be neglected.

The temperature stability of the high-Q resonators is important for its use in indoor localization tags. In harsh environments such as a fire in the building, indoor localization tag
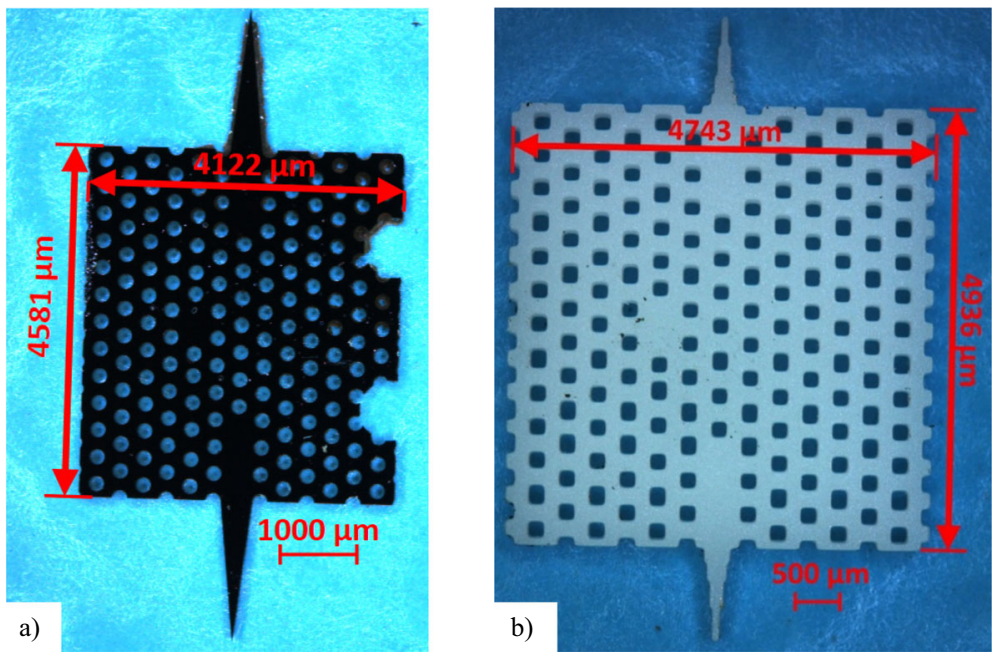

Fig. 9 a HR-Si and $\mathbf{b}$ alumina high-Q resonator sample at $230 \mathrm{GHz}$. In $\mathbf{b}$, the PhC holes have a square cross section to fit to the pixel raster of the $3 \mathrm{D}$ printer 

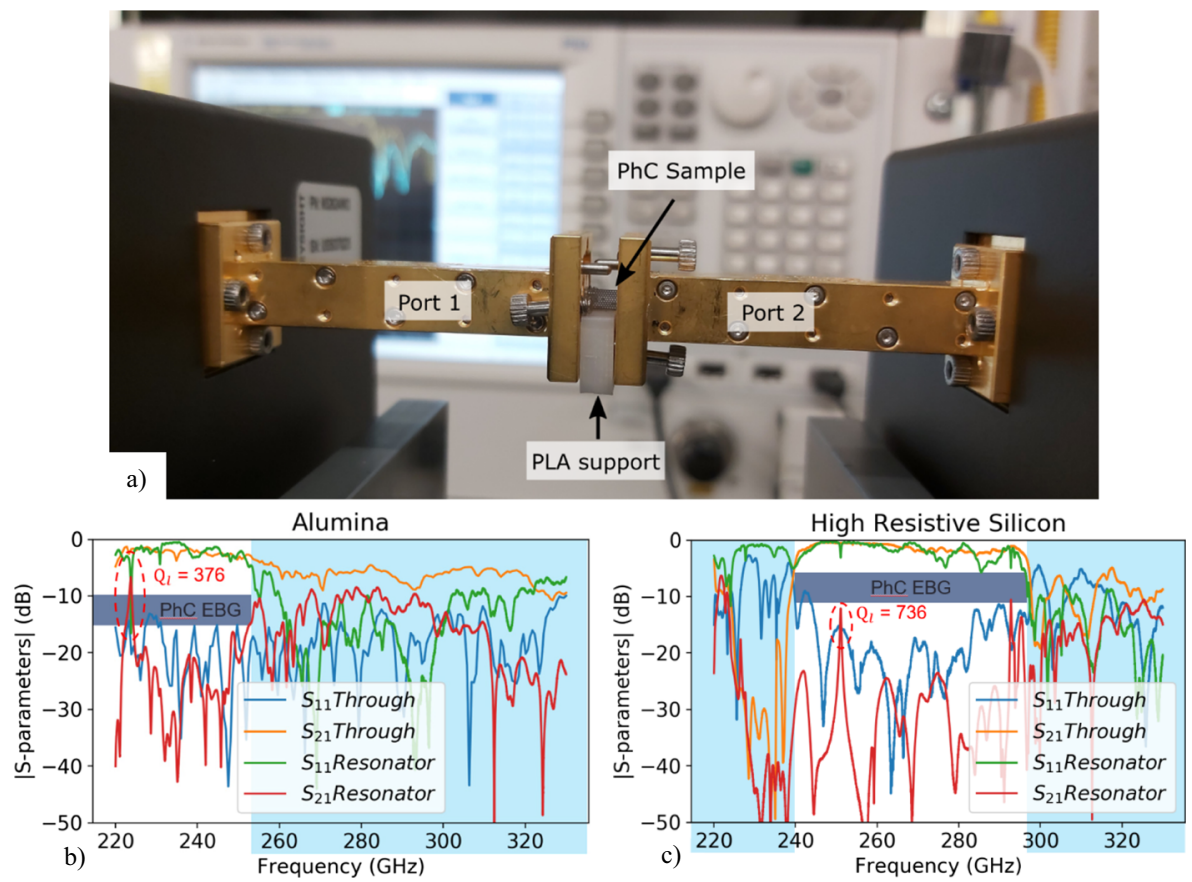

Fig. 10 a 2-port measurement setup for the characterization of the $230 \mathrm{GHz} \mathrm{PhCs}$. $\mathbf{b}$ and $\mathbf{c}$ show the results of the alumina and HR-Si resonator samples, respectively. In both cases, a direct connection (through) between the input and the output ports via a $\mathrm{PhC}$ dielectric waveguide is used as a reference. The extracted quality factors from the 3-dB bandwidth of the transmission peak for alumina and HR-Si are 376 and 736, respectively. EBG: electromagnetic bandgap corresponds to the operation frequencies of the $\mathrm{PhCs}$

landmarks able to withstand high temperatures on the ceiling would help unmanned vehicles to move through the building analyzing its state and the presence of humans in it. The temperature stability of the alumina and silicon high-Q resonators at $80 \mathrm{GHz}$ is shown in Fig. 11 [38]. $\mathrm{HR}-\mathrm{Si}$ presents lower losses at lower temperatures, but these losses rapidly increase with temperature and become higher than alumina for temperatures above $75{ }^{\circ} \mathrm{C}$. This increment of the losses reduces the quality factor needed for clutter suppression and also has a direct impact on the RCS as shown in Fig. 11b, reducing the maximum range.

In order to prove the feasibility of multi-resonator tags in a single $\mathrm{PhC}$, a 4-bit tag has been presented [39]. The RCS of a single resonator at $80 \mathrm{GHz}$ is $<-40 \mathrm{dBm}^{2}$ and the maximum range with a $25 \mathrm{dBi}$ standard gain horn antenna has been $20 \mathrm{~cm}$. Such 4-bit tag landmarks have been designed and measured at $230 \mathrm{GHz}$ with similar results. The manufactured samples in alumina and HR-Si and the measured 4 resonances can be seen in Fig. 12. The maximum readout range of the single-resonator 4-bit tag landmarks at $230 \mathrm{GHz}$ with a $25 \mathrm{dBi}$ reader antenna in the laboratory is $14 \mathrm{~cm}$. Using a metallic sphere with $2 \mathrm{~cm}$ diameter as a reference, an RCS of each resonator between $-55 \mathrm{dBm}^{2}$ and $-60 \mathrm{dBm}^{2}$ is obtained. Still, strong variations in the received power from each resonance are observed for different interrogation angles. This effect does not allow for the practical use of these tag landmarks unless a focusing structure such as waveguide or a lens is used to excite the resonators in a well-defined manner.

To increase maximum range of the high-Q resonator tags consistent with the localization system requirements, the RCS of single-resonator tags needs to be increased by at least 30$50 \mathrm{~dB}$. The RCS achieved by a single resonator can be increased with resonator arrays and 

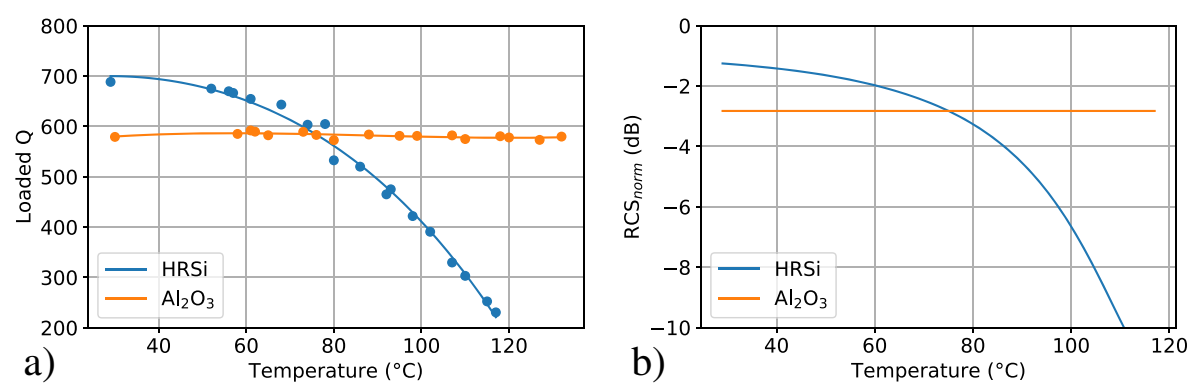

Fig. 11 a Temperature dependence of high-Q PhC resonators at $80 \mathrm{GHz}$ for alumina and HR-Si. b Its effect on the RCS of a high-Q resonator-based tag landmark for a radiation quality factor $Q \mathrm{rad}=1000$ [38]. $0 \mathrm{~dB}$ corresponds to a lossless case

corner reflectors. However, since all resonators contribute to the response, all of them should resonate exactly at the same frequency. The higher the Q-factor, the lower the tolerances of the system to frequency shifts which might result not only from different geometries of the resonators but also from variations in the permittivity of the different resonators. Even if low tolerance can be achieved, temperature gradients can shift the resonance frequency of some of the resonators which would lead to loss of functionality.

These limitations can be greatly reduced by using a lens such as a Luneburg lens to generate a focal area where the resonators are placed as shown in Fig. 13. In such a structure, only one or few resonators with the same resonance frequency are excited and their RCS is amplified by the lens. Since the interrogation propagates twice (in and out) of the Luneburg lens, the increase in the RCS of the resonators is twice the gain (in $\mathrm{dB}$ )

a)

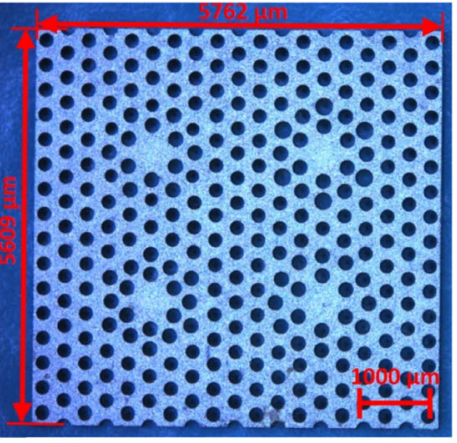

4-bit HR-Si tag

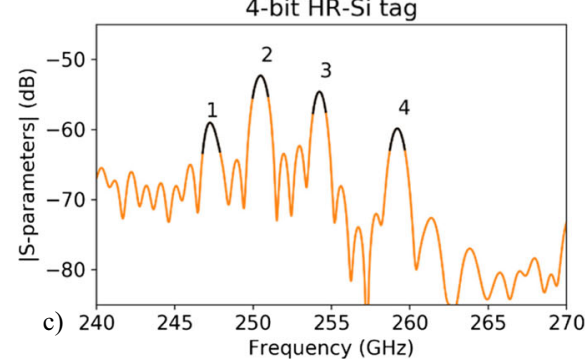

b)


Fig. 12 4-bit a alumina and $\mathbf{b}$ HR-Si tags. $\mathbf{c}$ and $\mathbf{d}$ show their frequency response at a distance $=15 \mathrm{~mm}$. In both cases, the four resonances can be differentiated as the 4 highest peaks 
Fig. 13 High-Q and high-RCS frequency-coded retro-reflector concept

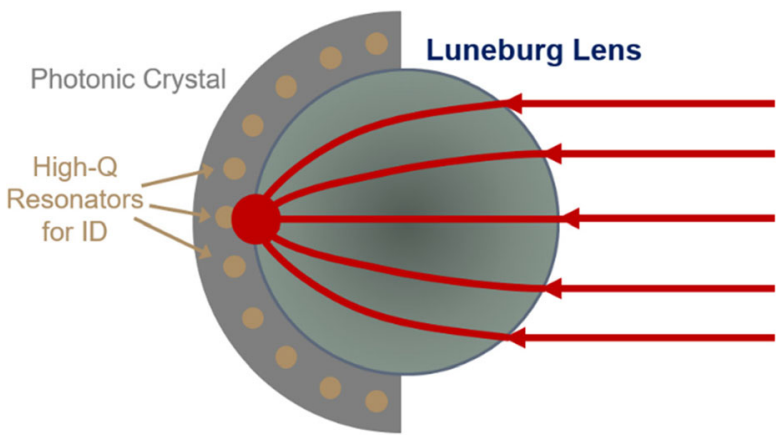

of such a lens placed in front of an antenna in a communication link. As shown in [13] using discrete DRs combined with single-index lens, by placing multiple resonators along the surface of the Luneburg lens, the coverage angle of a single tag can be widened, while only a few resonators are excited for each interrogation angle. While the information density that can be coded is still affected by shifts in the resonance frequencies of the resonators, its effects on the RCS or backscattered power of the tag are reduced. On the other hand, with intentionally varied resonance frequencies of the resonators, it should be possible to realize a passive chipless tag with angle-of-arrival sensing according to [16] with very fine angle resolution due to the narrow bandwidth of the high-Q resonators.

Examples of Luneburg lenses for communication links show gains of $24 \mathrm{~dB}$ at $90 \mathrm{GHz}$ [17] and $18 \mathrm{~dB}$ at $330 \mathrm{GHz}$ [40], to name a few examples. Therefore, an RCS increase of twice these gains, e.g., 36 to $48 \mathrm{~dB}$ can be expected. While such gains would potentially increase the range of the single dielectric resonators by a factor 8 to 16 , the fabrication and integration of multiple high-Q resonators within a lens at such high frequencies remains a challenge. A main issue is that the high relative permittivity $\epsilon_{\mathrm{r}}>9$ needed for the efficient realization of high-Q resonators diverges from the low permittivity gradient from 1 to 2 required for the conventional Luneburg lens.

\section{Conclusion}

This paper has presented the evolution of tag landmark designs for a novel selflocalization system. Tags based on single dielectric resonators were found to provide retro-directive scattering but with far too low RCS levels for our system application. A tag based on low-Q resonators and aimed at very high RCS levels was found to be best realized in the form of corner reflectors which include large planar arrays of dielectric resonators to create notched spectral signatures of the monostatic RCS. These designs can be scaled in size to realize any required RCS level and the position of the spectral notch can be defined by the choice of diameter of the dielectric resonators. Due to the wide-band high RCS characteristic, this tag design provides very high range resolution while frequency position coding allows only few bits and the read range can be limited by the typical corner reflector wide-angle degradation. 
A tag based on high-Q resonators is envisaged in a design combining planar photonic crystal resonators with a spherical Luneburg lens. Due to the smaller resonance bandwidths and the combination of several resonators in one tag, this design promises much larger coding range and due to the spherical lens, a much wider angular coverage at constant high RCS level.

With view to a practical tag manufacture for the lower THz frequency range, both concepts can profit from 3D printing technologies for ceramic materials, as in [39], which allows fabrication of ceramic resonators in planar arrays, photonic crystals, or other planar and nonplanar configurations without, e.g., the requirement of manual placement of single resonators.

Funding Information Open Access funding provided by Projekt DEAL. This work was funded by the Deutsche Forschungsgemeinschaft (DFG, German Research Foundation) - Project-ID 287022738 - TRR 196 within projects $\mathrm{C} 09, \mathrm{~S} 04, \mathrm{C} 12$, and $\mathrm{C} 13$.

Open Access This article is licensed under a Creative Commons Attribution 4.0 International License, which permits use, sharing, adaptation, distribution and reproduction in any medium or format, as long as you give appropriate credit to the original author(s) and the source, provide a link to the Creative Commons licence, and indicate if changes were made. The images or other third party material in this article are included in the article's Creative Commons licence, unless indicated otherwise in a credit line to the material. If material is not included in the article's Creative Commons licence and your intended use is not permitted by statutory regulation or exceeds the permitted use, you will need to obtain permission directly from the copyright holder. To view a copy of this licence, visit http://creativecommons.org/licenses/by/4.0/.

\section{References}

1. W. Sakperea, M. Adeyeye-Oshinb and N. B. W. Mlitwac, "A State-of-the-Art Survey of Indoor Positioning and Navigation Systems and Technologies," South African Computer Journal, vol. 29, no. 3, p. 145-197, 2017.

2. H. Liu, H. Darabi, P. Banerjee and J. Liu, "Survey of wireless indoor positioning techniques and systems," IEEE Transactions on Systems, Man, and Cybernetics, Part C(Applications and Reviews), vol. 37, no. 6, pp. 1067-1080, 2007.

3. O. Kanhere and T. S. Rappaport, "Position locationing for millimeter wave systems," in Proceedings of 2018 IEEE Global Communications Conference (GLOBECOM), 2018.

4. J. Kokert, F. Höflinger and L. M. Reindl, "Indoor localization system based on galvanometer-laser-scanning for numerous mobile tags (GaLocate)," in 2012 International Conference on Indoor Positioning and Indoor Navigation (IPIN), Sydney, 2012.

5. N. Pohl, T. Jaeschke, S. Küppers, C. Bredendiek and D. Nüßler, “A Compact Ultra-Wideband mmWave Radar Sensor at $80 \mathrm{GHz}$ based on a SiGe Transceiver Chip," in IEEE 22nd International Microwave and Radar Conference (MIKON), 2018.

6. N. Pohl, T. Jaeschke and M. Vogt, "An SiGe-chip-Based $80 \mathrm{GHz}$ FMCW-radar system with $25 \mathrm{GHz}$ bandwidth for high resolution imaging," in 14th International Radar Symposium (IRS), 2013.

7. M. El-Absi, A. Alhaj Abbas, A. Abuelhaija, F. Zheng, K. Solbach and T. Kaiser, "High-accuracy indoor localization based on Chipless RFID systems at THz band," IEEE Access, vol. 6, pp. 54355-54,368, 2018.

8. C. Mandel, B. Kubina, M. Schüßler and R. Jakoby, "Metamaterial inspired passive chipless radio-frequency identification and wireless sensing," Annals of Telecommunications, vol. 68, no. 7-8, pp. 385-399, 2013.

9. C. Mandel, B. Kubina, M. Schüßler, R. Jakoby, G. vom Bögel, F. Meyer, A. Grabmaier, M. Wiemeler, T. Kaiser and K. Solbach, "Approach for long-range frequency domain chipless RFID tags towards THz," in Proc. Smart SysTech, Duisburg, Germany, 2016.

10. A. Alhaj Abbas, A. Abuelhaija and K. Solbach, "Investigation of the transient EM scattering of a dielectric resonator," in 11th German Microwave Conference (GeMiC), pp. 271-274, Mar. 2018.

11. M. Gastine, L. Courtois and J. Dormann, "Electromagnetic Resonances of Free Dielectric Spheres," IEEE Transactions on Microwave Theory and Techniques, vol. 15, no. 12, pp. 694-700, 1967.

12. A. Alhaj Abbas, M. El-Absi, A. Abuelhaija, K. Solbach and T. Kaiser, "RCS enhancement of dielectric resonator tag using spherical lens," Frequenz, vol.73, no.5-6, pp. 161-170, 2019. 
13. A. Alhaj Abbas, M. El-Absi, A. Abuelhaija, K. Solbach and T. Kaiser, "Wide-Angle RCS Enhanced Tag Based on Dielectric Resonator - Lens Combination," Frequenz, vol.74, no.1-2, pp. 1 - 8, 2020.

14. A. Alhaj Abbas, M. El-Absi, A. Abuelhaija, K. Solbach and T. Kaiser, "High RCS Passive Tag based on Dielectric Resonator - 2D Lens Combination," in 12th German Microwave Conference (GeMiC), 2019.

15. Y. Zhao, J. Weidemueller, G. Vom Bögel, A. Grabmaier, A. Alhaj Abbas, K. Solbach, A. Jiménez-Sáez, M. Schüßler and R. Jakoby, "2D Luneburg Lens Made of Metamaterial for Chipless Dielectric Resonator Tags in the Localization Application," in 2019 International Workshop on Mobile THz Systems, Bad Neuenahr, Germany, July 2019.

16. A. Alhaj Abbas, M. El-Absi, A. Abuelhaija, K. Solbach and T. Kaiser, "Dielectric Resonator-Based Passive Chipless Tag with Angle-of-Arrival Sensing," IEEE Transactions on Microwave Theory and Techniques, vol. 67, no. 5, pp. 2010-2017, 2019.

17. Z. Larimore, S. Jensen, A. Good, A. Lu, J. Suarez and M. Mirotznik, "Additive manufacturing of Luneburg lens antennas using space-filling curves and fused filament fabrication," IEEE Transactions on Antennas and Propagation, vol. 66, no. 6, pp. 2818-2827, 2018.

18. A. Trubin, Lattices of Dielectric Resonators, Springer, 2016.

19. A. Alhaj Abbas, M. El-Absi, A. Abuelhaija, K. Solbach and T. Kaiser, "Terahertz Passive RFID Tag Based on Dielectric Resonator Linear Array," in 2019 International Workshop on Mobile THz Systems, Bad Neuenahr, Germany, July 2019.

20. M. El-Absi, A. Alhaj Abbas, A. Abuelhaija, K. Solbach and T. Kaiser, "Chipless RFID Infrastructure Based Self-Localization: Testbed Evaluation," in IEEE Veh. Tech., accepted for publication, 2020.

21. A. Alhaj Abbas, M. El-Absi, A. Abuelhaija, K. Solbach and T. Kaiser, "Metallic Reflectors with Notched RCS Spectral Signature using Dielectric Resonators," IET Electronics Letters, vol. 56, no. 6, pp. 273-276, 2020 .

22. J. Barowski, A. Alhaj Abbas, M. El-Absi, L. Piotrowsky, N. Pohl, I. Rolfes and K. Solbach, "Design and Evaluation of a Passive Frequency-Coded Reflector using W-Band FMCW Radar," in German Microwave Conference (GeMiC), Cottbus, Germany, March 2020.

23. A. Alhaj Abbas, M. El-Absi, A. Abuelhaija, K. Solbach and T. Kaiser, "Corner Reflector with RCS Frequency Coding by Dielectric Resonators," IET Microwaves, Antennas \& Propagation, revision submitted, May 2020.

24. K. Solbach, A. Alhaj Abbas, M. El-Absi, A. Abuelhaija and T. Kaiser, "Experimental Demonstration of Double-Notch RCS Spectral Signature of Corner Reflector Beacon for THz Self-Localization System," in International Workshop on Mobile THz Systems (IWMTS2020), Essen, Germany, 2020.

25. A. Jiménez-Sáez, M. Schüßler, M. El-Absi, A. Alhaj Abbas, K. Solbach, T. Kaiser and R. Jakoby, "Frequency Selective Surface Coded Retroreflectors for Chipless Indoor Localization Tag Landmarks," IEEE Antennas and Wireless Propagation Letters, vol.19, no.5, pp. 726-730, 2020.

26. D. Thomson, D. Card and G. Bridges, "RF cavity passive wireless sensors with time-domain gating-based interrogation for SHM of civil structures," IEEE Sensors Journal, vol. 9, no. 11, pp. 1430-1438, 2009.

27. B. Kubina, M. Schüßler, C. Mandel, A. Mehmood and R. Jakoby, "Wireless high-temperature sensing with a chipless tag based on a dielectric resonator antenna," in 2013 IEEE Sensors, Baltimore, MD, pp. 1-4, 2013.

28. A. Jiménez-Sáez, E. Polat, C. Mandel, M. Schüßler, B. Kubina, T. Scherer, N. Lautenschläger and R. Jakoby, "Chipless wireless temperature sensor for machine tools based on a dielectric ring resonator," in Procedia Engineering 168, pp. 1231-1236, 2016.

29. J. Molla, R. Heidinger, A. Ibarra and G. Link, "Dielectric properties of alumina/zirconia composites at millimeter wavelengths," Journal of applied physics, vol. 73, no. 11, pp. 7667-7671, 1993.

30. S. M. Hanham, M. M. Ahmad, S. Lucyszyn and N. Klein, "LED-switchable high-Q packaged THz microbeam resonators," IEEE Transactions on Terahertz Science and Technology, vol. 7, no. 2, pp. 199208, 2017.

31. T. Tanabe, M. Notomi, E. Kuramochi, A. Shinya and H. Taniyama, "Trapping and delaying photons for one nanosecond in an ultrasmall high-Q photonic-crystal nanocavity," Nature Photonics, vol. 1, no. 1, pp. 4952, 2007.

32. H. Altug, D. Englund and J. Vučković, "Ultrafast photonic crystal nanocavity laser," Nature physics, vol. 2, no. 7, pp. $484-488,2006$.

33. R. Leijssen and E. Verhagen, "Strong optomechanical interactions in a sliced photonic crystal nanobeam," Scientific reports, vol. 5, no. 1, pp. 1-10, 2015.

34. H. Pier, E. Kapon and M. Moser, "Strain effects and phase transitions in photonic resonator crystals," Nature, vol. 407, no. 6806, pp. 880-883, 2000.

35. K. Okamoto, K. Tsuruda, S. Diebold, S. Hisatake, M. Fujita and T. Nagatsuma, "Terahertz sensor using photonic crystal cavity and resonant tunneling diodes," Journal of Infrared, Millimeter, and Terahertz. Waves, vol. 38, no. 9, pp. 1085-1097, 2017. 
36. S. Hanham, C. Watts, W. Otter, S. Lucyszyn and N. Klein, "Dielectric measurements of nanoliter liquids with a photonic crystal resonator at terahertz frequencies," Applied Physics Letters, vol. 107, no. 3, 2015.

37. A. Jiménez-Sáez, M. Schüßler, C. Krause, D. Pandel, K. Rezer, G. Vom Bögel, N. Benson and R. Jakoby, "3D printed alumina for low-loss millimeter wave components," IEEE Access, vol. 7, pp. 40719-40,724, 2019.

38. A. Jiménez-Sáez, M. Schüßler, D. Pandel, C. Krause, Y. Zhao, G. vom Bögel, N. Benson and R. Jakoby, "Temperature Characterization of High-Q Resonators of Different Materials for mm-Wave Indoor Localization Tag Landmarks," in 15th European Conference on Antennas and Propagation, 2020.

39. A. Jiménez-Sáez, M. Schüßler, D. Pandel, N. Benson and R. Jakoby, "3D Printed 90 GHz FrequencyCoded Chipless Wireless RFID Tag," in 2019 IEEE MTT-S International Microwave Workshop Series on Advanced Materials and Processes for RF and THz Applications (IMWS-AMP), 2019.

40. D. Headland, W. Withayachumnankul, R. Yamada, M. Fujita and T. Nagatsuma, "Terahertz multi-beam antenna using photonic crystal waveguide and Luneburg lens," APL Photonics, vol. 3, no. 12, Dec. 2018.

Publisher's Note Springer Nature remains neutral with regard to jurisdictional claims in published maps and institutional affiliations.

\section{Affiliations}

\section{A. Jiménez-Sáez ${ }^{1}$ - A. Alhaj-Abbas ${ }^{2}$ - M. Schüßler ${ }^{1}$ - A. Abuelhaija ${ }^{2,3} \cdot$ M. El-Absi ${ }^{2} \cdot$ M.}

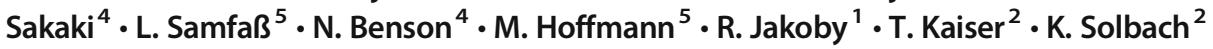

Institute of Microwave Engineering and Photonics, Technische Universität Darmstadt, Darmstadt, Germany

2 Institute of Digital Signal Processing, Universität Duisburg-Essen, Duisburg, Germany

3 Applied Science Private University, Amman, Jordan

4 Institute of Technology for Nanostructures, Universität Duisburg-Essen, Duisburg, Germany

5 Ruhr Universität Bochum, Bochum, Germany 